Check for updates

Cite this: RSC Adv., 2017, 7, 29051

Received 4th April 2017

Accepted 24th May 2017

DOI: $10.1039 / \mathrm{c} 7 \mathrm{ra03848h}$

rsc.li/rsc-advances

\title{
A novel three-dimensional hierarchical CuAl layered double hydroxide with excellent catalytic activity for degradation of methyl orange
}

\author{
Jialun Li, ${ }^{a}$ Shuhua Zhang, (D) *a Yang Chen, ${ }^{a}$ Tianmeng Liu, ${ }^{a}$ Chunyan Liu, ${ }^{b}$ Xu Zhang, ${ }^{a}$ \\ Mengting $\mathrm{Yi}^{\text {a }}$ Zhongyang $\mathrm{Chu}^{\mathrm{a}}$ and Xiuxiu Han ${ }^{\mathrm{a}}$
}

\begin{abstract}
Heterogeneous fenton-like degradation of methyl orange $(\mathrm{MO})$ in water was investigated over CuAlLayered Double Hydroxide (CUAl-LDH) with high copper ions content prepared by hydrothermal synthesis in dimethyl sulfoxide-water mixed solvent. CuAl-LDH was proved to have a novel spherical morphology with complex secondary structures and possessed very high crystallinity and purity by XRD, FT-IR, EA, ICP, TEM and SEM characterization. The degradation of MO on this material was evaluated via examining catalyst loading, hydrogen peroxide dosage and temperature when the reaction temperature was $40{ }^{\circ} \mathrm{C}$, the dosage of $\mathrm{LDH}$ and $\mathrm{H}_{2} \mathrm{O}_{2}$ were $20 \mathrm{mg}$ and $0.5 \mathrm{~mL}$, respectively. These results indicated the obtained CuAl-LDH exhibited an excellent performance for oxidation degradation of $\mathrm{MO}$ by comparing with MgAl-LDH and CuMgAl-LDH, which will be efficient for the practical application in the abatement of organic pollutants.
\end{abstract}

\section{Introduction}

With the rapid development of urbanization and industrialization, many countries have been confronted with a serious crisis of water resources, and the problem of water pollution has intensified. ${ }^{1}$ As a member of the azo dyes, MO was widely used in the textile processing, paper making, printing and cosmetic industries. ${ }^{2}$ During the dyeing process, a mass of industrial effluents containing dyes were released into the environment, which has been attracting serious public concern due to their intense color, toxicity, carcinogenicity and mutagenicity. ${ }^{3}$ However, it is the complex molecular structure and low degradability that make the dye-containing effluents more and more difficult to treat. The conventional processes such as adsorption, coagulation, and membrane separation have hardly achieved a good effect. ${ }^{4}$ Therefore, it is necessary to develop effective methods to treat MO wastewater. Nowadays, more and more new technologies have been developed to decompose organic pollutants, such as carbon-based catalysts, ${ }^{5,6}$ photo catalysts, $^{7}$ electrocatalysts ${ }^{8-10}$ and advanced oxidation processes, ${ }^{11,12}$ which offer attractive alternatives to traditional methods for treating wastewaters containing organic compounds.

${ }^{a}$ College of Chemistry and Chemical Engineering, Shanghai University of Engineering Science, Shanghai 201620, China. E-mail: zsh_7474@126.com; zhangsh@sues.edu. cn; Tel: +86-13916737957

${ }^{b}$ College of Mechanical Engineering, Shanghai University of Engineering Science, Shanghai 201620, China
Among the different advanced oxidation processes, the heterogeneous Fenton process has been found to be very effective in the degradation of organic pollutants. ${ }^{13,14}$ In this process, hydrogen peroxide is decomposed into hydroxyl radicals with the help of a heterogeneous catalyst, which can convert a large range of hazardous organic pollutants into easily biodegradable intermediate species or even carbon dioxide, water and inorganic salts, due to their high reactivity and low selectivity. ${ }^{15,16}$ However, the application of the classic iron-based Fenton process has been limited by its tight $\mathrm{pH}$ range, and in order to avoid a large amount of iron sludge being produced, strict acidic conditions (below $\mathrm{pH} 4$ ) are required. ${ }^{17}$ Thus, to overcome these drawbacks, the current study is focused on finding a heterogeneous catalyst which can work over a higher $\mathrm{pH}$ range. A variety of catalysts containing $\mathrm{Cu}$ have been researched, and it has already been confirmed that using $\mathrm{Cu}^{-}$ based catalysts to undergo a Fenton-type reaction can efficiently oxidize various organic pollutants in neutral aqueous solutions. ${ }^{18}$

Layered double hydroxides (LDHs) have been receiving enormous attention in the field of heterogeneous catalysis due to their outstanding catalytic behaviors, such as activity, selectivity and stability of active sites. ${ }^{19-22}$ In particular, the coppercontaining LDHs with the extremely high catalytic activity of copper ions show excellent catalytic performances for VOC oxidation and organic dye degradation, as well as phenol hydroxylation. ${ }^{23,24}$ S. Kannan and coworkers synthesized CuMgAl ternary hydrotalcites as catalysts for the hydroxylation of phenol by coprecipitation. ${ }^{25}$ Jean Fahel and coworkers investigated the CoCuAl-LDH which exhibited excellent catalytic 
activity on the dye degradation of orange $\mathrm{II} .{ }^{26}$ Hongtao Lu and coworkers prepared and applied CuZnFe-LDH as a heterogeneous Fenton-like oxidation catalyst for the degradation of acetaminophen. ${ }^{27}$ Meanwhile, Cu-containing derivatives of have been widely studied. Guoqing Cui and coworkers prepared $\mathrm{CuMgAl}-\mathrm{LDH} @ \mathrm{SiO}_{2}$ nanosheets by delaminating $\mathrm{CuMgAl}\left(\mathrm{NO}_{3}\right)-\mathrm{LDH}$ microcrystals followed by coating with a layer of $\mathrm{SiO}_{2}$, which increased the amount of exposed active sites and remarkably enhanced catalytic activity in phenol hydroxylation. ${ }^{28}$ Hui Zhang and coworkers reported a facile onepot coprecipitation assembly of a hierarchical core@shell structured $\mathrm{Fe}_{3} \mathrm{O}_{4} @ \mathrm{CuMgAl}-\mathrm{LDH}$ magnetic nanocatalyst exhibiting higher catalytic activity for phenol hydroxylation by $\mathrm{H}_{2} \mathrm{O}_{2}$ than pure CuMgAl-LDH. ${ }^{29}$ Although ternary LDHs with a hierarchical flower-like structure with introduced $\mathrm{Cu}^{2+}$ species enhanced the catalytic activity on catalytic degradation of organic pollutants, the content of $\mathrm{Cu}^{2+}$ was quite low. It has been reported that $\mathrm{Cu}$-containing LDHs show good catalytic activity, which is attributable to the content of $\mathrm{Cu}^{2+}$ active sites and the better dispersion of copper ions. ${ }^{30}$ Therefore, some efforts have recently been made to synthesize binary $\mathrm{Cu}$ containing LDHs. Although many kinds of $\mathrm{Cu}-\mathrm{Al}$ type hydrotalcites have been successfully synthesized, it is hard to obtain a single $\mathrm{Cu}-\mathrm{LDH}$ crystal with high $\mathrm{Cu}^{2+}$ content, because it is always mixed with impurity phases, such as malachite or gerhardtite, because of the Jahn-Teller effect. ${ }^{31,32}$ What is more, the morphology is no longer a flower-like structure composed of nanosheets, but is reunited with the block structure.

In this work, a 3D hierarchical flower-like CuAl-layered double hydroxide has been successfully prepared via a simple one-step solvothermal method. The as-prepared samples were synthesized solvothermally in dimethyl sulfoxide-water mixed solvents. This not only keeps the solution at a higher polarity, but also provides suitable pressure and $\mathrm{pH}$ for the formation of layered double hydroxides. Compared with the conventional binary or ternary $\mathrm{Cu}$-containing LDHs, it has higher purity and crystallinity. This could be explained by the fact that dimethyl sulfoxide possesses strong water imbibition, resulting in an increase in the concentration of urea, which eases the hydrolysis of urea. In the meantime, this process also leads to a slow increase in the $\mathrm{pH}$ of the solution and maintains it at a relatively low value $(\sim 5.2)$, which not only satisfies the need for LDHs nucleation and growth, but also inhibits the formation of various impurities. The as-synthesized binary CuAl-LDH possesses a delicate structure, good crystallinity, and higher copper content, and displays excellent sensitivity and selectivity for the degradation of methyl orange dye pollutants.

\section{Materials and methods}

\subsection{Chemicals}

$\mathrm{Cu}\left(\mathrm{NO}_{3}\right)_{2} \cdot 3 \mathrm{H}_{2} \mathrm{O}, \quad \mathrm{Al}\left(\mathrm{NO}_{3}\right)_{3} \cdot 9 \mathrm{H}_{2} \mathrm{O}, \mathrm{Mg}\left(\mathrm{NO}_{3}\right)_{2} \cdot 6 \mathrm{H}_{2} \mathrm{O}$ and urea were all of reagent grade and were purchased from Sinopharm Chemical Reagent Co., Ltd., China. Dimethyl sulfoxide (DMSO), $\mathrm{NaCO}_{3}$ and $\mathrm{NaOH}$ were obtained from the Sigma-Aldrich Chemical Company. All reagents were used directly without further purification.

\subsection{Equipment}

The crystal structures of the samples were determined by X-ray diffraction (D2 Phaser, Bruker, Germany) at $35 \mathrm{kV}$ and $30 \mathrm{~mA}$, using $\mathrm{Cu} \mathrm{K} \alpha$ radiation in the $2 \theta$ range of $5-80^{\circ}$. The surface functional groups for $\mathrm{CuAl}-\mathrm{LDH}$ were detected by Fourier transform infrared spectrometry (FT-IR) (AVATAR380, Nicolet, USA). The morphology and microstructures of the samples were characterized by scanning electron microscopy (SEM, JEOL4800) and transmission electron microscopy (TEM, JEOL1011), respectively. $\mathrm{N}_{2}$ adsorption-desorption isotherms were determined on a Micromeritics ASAP 2460 instrument from which the surface area $\left(S_{\mathrm{BET}}\right)$, pore volume $\left(V_{\mathrm{P}}\right)$ and pore diameter $\left(D_{\mathrm{P}}\right)$ were calculated by applying the BrunauerEmmett-Teller (BET) and Barrett-Joyner-Halenda (BJH) models on the desorption branches. The UV-vis spectra of the materials were recorded in the $200-800 \mathrm{~nm}$ range using a UV-visible spectrometer (UV-1900). The compositions of the samples were analyzed by inductively coupled plasma analysis (ICP) using a Varian 725-ES device and a Euro Elemental Analyser.

\subsection{Preparation of Cu-Al LDH, MgAl-LDH and MgAlCu- LDH}

CuAl-LDH was synthesized by a hydrothermal method. In a typical synthesis, $9 \mathrm{mmol}$ of $\mathrm{Cu}\left(\mathrm{NO}_{3}\right)_{2} \cdot 3 \mathrm{H}_{2} \mathrm{O}$ and $4.5 \mathrm{mmol}$ of $\mathrm{Al}\left(\mathrm{NO}_{3}\right)_{3} \cdot 9 \mathrm{H}_{2} \mathrm{O}$ were dissolved in a mixture of $45 \mathrm{~mL}$ of deionized water and $15 \mathrm{~mL}$ of dimethyl sulfoxide, and then, the urea was directly added into the above solution at $0.2 \mathrm{~mol} \mathrm{~L}^{-1}$. The mixed solutions were stirred continuously for $10 \mathrm{~min}$ to form a transparent solution. Afterward, the as-obtained homogeneous solution was transferred to a $100 \mathrm{~mL}$ Teflon-lined stainless-steel autoclave and heated at $110{ }^{\circ} \mathrm{C}$ for $12 \mathrm{~h}$. The obtained precipitate was filtered, washed with distilled water and ethanol several times, and then dried at $100 \pm 2{ }^{\circ} \mathrm{C}$ for $12 \mathrm{~h}$, to produce $\mathrm{CuAl}-\mathrm{NO}_{3} \cdot \mathrm{H}_{2} \mathrm{O}$ HT powder.

The MgAl-LDH and CuMgAl-LDH were prepared by coprecipitation. A mixed basic solution containing $\mathrm{NaOH}$ and $\mathrm{Na}_{2} \mathrm{CO}_{3}$ with solution concentrations of $0.01 \mathrm{~mol} \mathrm{~L}^{-1}$ and $0.005 \mathrm{~mol} \mathrm{~L}^{-1}$, respectively, and mixed salt solutions containing $0.03 \mathrm{~mol} \mathrm{~L}^{-1} \mathrm{Mg}\left(\mathrm{NO}_{3}\right)_{2} \cdot 6 \mathrm{H}_{2} \mathrm{O}$ and $0.01 \mathrm{~mol} \mathrm{~L}^{-1} \mathrm{Al}\left(\mathrm{NO}_{3}\right)_{2} \cdot 9 \mathrm{H}_{2} \mathrm{O}$ were simultaneously added dropwise into the deionized water at a speed of 1 drop per s, under vigorous stirring at $60{ }^{\circ} \mathrm{C}$. The mixture was stirred for $4 \mathrm{~h}$ with a constant $\mathrm{pH}=10$. The precipitate was then filtered and washed with deionized water, and dried at $80{ }^{\circ} \mathrm{C}$ for $10 \mathrm{~h}$. CuMgAl-LDH was obtained via the same strategy with the designed molar ratio $\mathrm{Mg}: \mathrm{Al}: \mathrm{Cu}$ of $3: 1: 1$.

\subsection{Catalytic reaction}

The catalytic reaction was carried out in a $50 \mathrm{~mL}$ beaker and heated in a water bath under atmospheric pressure. In a typical run, a given dosage of CuAl-LDHs and $\mathrm{H}_{2} \mathrm{O}_{2}$ was added to 50 $\mathrm{mL}$ of $20 \mathrm{mg} \mathrm{L}^{-1}$ aqueous methyl orange solution at a certain temperature. In order to study the effect of HTs on the degradation reaction, the experiments were repeated under different catalyst loadings, $\mathrm{H}_{2} \mathrm{O}_{2}$ dosages and temperatures. The amount 
of catalyst used was in the range of $0-40 \mathrm{mg}$ and the added amount of $\mathrm{H}_{2} \mathrm{O}_{2}$ was in the range of $0-2 \mathrm{~mL}$, with an initial $\mathrm{pH}$ of near-neutral conditions from $25{ }^{\circ} \mathrm{C}$ to $40{ }^{\circ} \mathrm{C}$. When the optimum reaction conditions were determined, some control experiments were comparatively conducted to demonstrate the advantage of the as-synthesized catalyst compared to other types of $\mathrm{LDH}$ materials. During the reactions, $1 \mathrm{~mL}$ of the reaction solution was withdrawn by a syringe every 10 minutes and immediately filtered through a Millipore filter to remove the solid particles. Subsequently, the concentration of methyl orange was measured by UV-vis spectroscopy. After the reaction, the catalyst was collected by centrifuging and washed with distilled water several times. After drying, the recycled catalyst was reused in the next run under the same conditions. The degradation efficiency of the dye was calculated from the equation:

$$
\text { Degradation efficiency }=\frac{A_{0}-A_{t}}{A_{0}} \times 100 \%
$$

where $A_{0}$ was the initial absorbance and $A_{t}$ was the absorbance at time $t$.

\section{Results and discussion}

\subsection{Characterization results analysis}

The XRD spectrum of the as-prepared sample is shown in Fig. 1. There are sharp and symmetrical reflections at $9.94^{\circ}, 20.04^{\circ}$, $30.24^{\circ}$, which correspond to diffractions of the (003), (006) and (009) planes in the layered structure, respectively. Broader, less intense reflections at $34.59^{\circ}, 59.82^{\circ}, 60.31^{\circ}$ also appeared due to the diffraction of the (015), (110) and (113) planes, confirming the formation of a well-crystallized hydrotalcite structure with $R 3 m$ rhombohedral symmetry. ${ }^{33,34}$ The diffraction peaks of the as-obtained samples are sharp and intense, and no impurity peaks are observed, indicating the highly crystalline nature and high purity of the sample. However, the orderliness of the layer shows a clean decline with increasing copper content, as indicated by the decrease in both intensity and sharpness of the (110) and (113) reflections, due to the Jahn-Teller distortion caused by poor long-range ordering. ${ }^{35}$ Furthermore, we find that there is an obvious positional shift of the (00l) diffraction

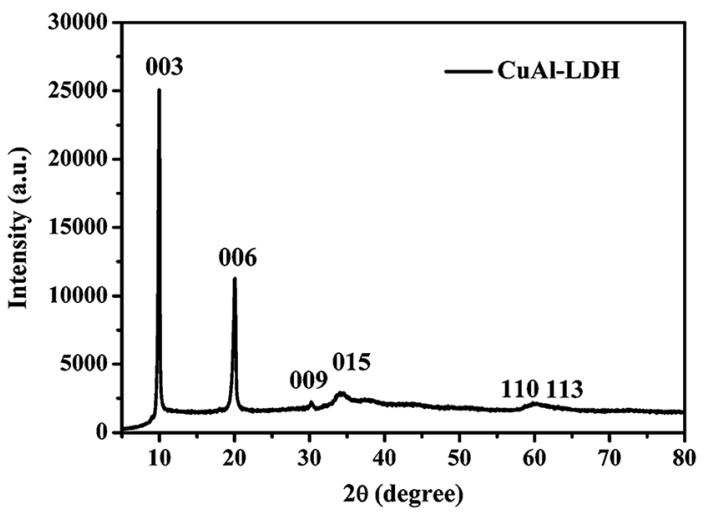

Fig. 1 XRD diffraction pattern of CuAl-LDH. toward low $2 \theta$ angles, denoting an increase in the basal spacing. Lattice parameters $a$ and $c$, calculated from the (003), (006), and (110) reflections, are $3.09 \AA$ and $26.6 \AA$, respectively. In general, there are two reasons for the increase. The first is assigned to the nitrate ion, which is not only larger than the carbonate ion, but also has a lower charge. Usually, the lower the charge density in the layers or the larger the effective radius of the interlayer anions, the larger the lattice parameter $c .{ }^{36}$ The second is assigned to the Jahn-Teller distortion in the $\mathrm{Cu}^{2+}$ ions resulting in an elongated octahedron.

The FT-IR spectrum of the as-synthesized LDH is shown in Fig. 2. The broad and strong adsorption peak at about 3382 $\mathrm{cm}^{-1}$ can be ascribed to the $\mathrm{OH}$-stretching vibration in the brucite-like layers and interlayer water, accompanied with the bending mode at $1636 \mathrm{~cm}^{-1} \cdot{ }^{37}$ In addition, the intense absorption peak at $1340 \mathrm{~cm}^{-1}$ corresponds to the $v_{3}$ vibration mode of interlayer carbonate anions. Meanwhile, two weak bands at 1051 and $816 \mathrm{~cm}^{-1}$ are also observed due to the $\mathrm{v}_{1}$ and $\mathrm{v}_{2}$ vibrations of $\mathrm{CO}_{3}{ }^{2-}$ ions. ${ }^{38}$ In the low-frequency region, the peak at $753 \mathrm{~cm}^{-1}$ results from the lattice vibration of $\mathrm{M}-\mathrm{O}$, $\mathrm{M}-\mathrm{O}-\mathrm{M}$ and $\mathrm{O}-\mathrm{M}-\mathrm{O}$ species in the layers. ${ }^{39}$ However, the $\mathrm{v}_{2}$ and $\mathrm{v}_{4}$ vibrations of carbonate shift toward lower wave numbers as the copper content increases, which is probably due to the Jahn-Teller distortion of the $\mathrm{Cu}(\mathrm{OH})_{6}$ octahedra, leading to an elongation in the $c$-axis, thus facilitating hydrogen bonding with interlayer carbonate anions. ${ }^{\mathbf{4 0}}$

The SEM/TEM images of the obtained samples are shown in Fig. 3. The CuAl-LDH possesses an almost flower-like morphology, which is caused by many irregular and randomly arranged nanosheets, exhibiting layer structures with crystal dispersion. In Fig. 3b a single flower-like microsphere can be further observed. These flexible nanoplates wrap round and connect to each other and are assembled perpendicularly to the spherical surface with clearly oriented layers. Additionally, a large amount of interconnected networks of macropores exists in the CuAl-LDH samples between the layers and the hierarchical structure. The TEM also corroborates the less agglomerated porous platelet structure for CuAl-LDHs. It can be seen that the nanosheets are self-assembled to form flower-like nanostructures, which is consistent with the SEM results.

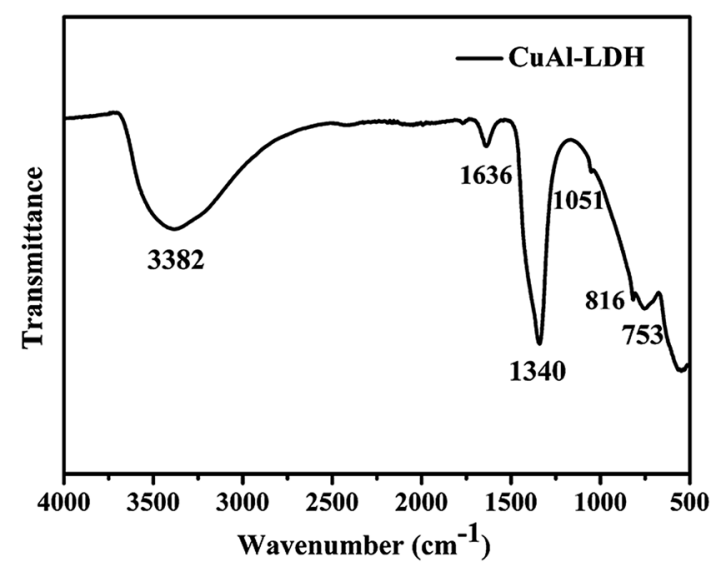

Fig. 2 FT-IR spectrum of CuAl-LDH. 


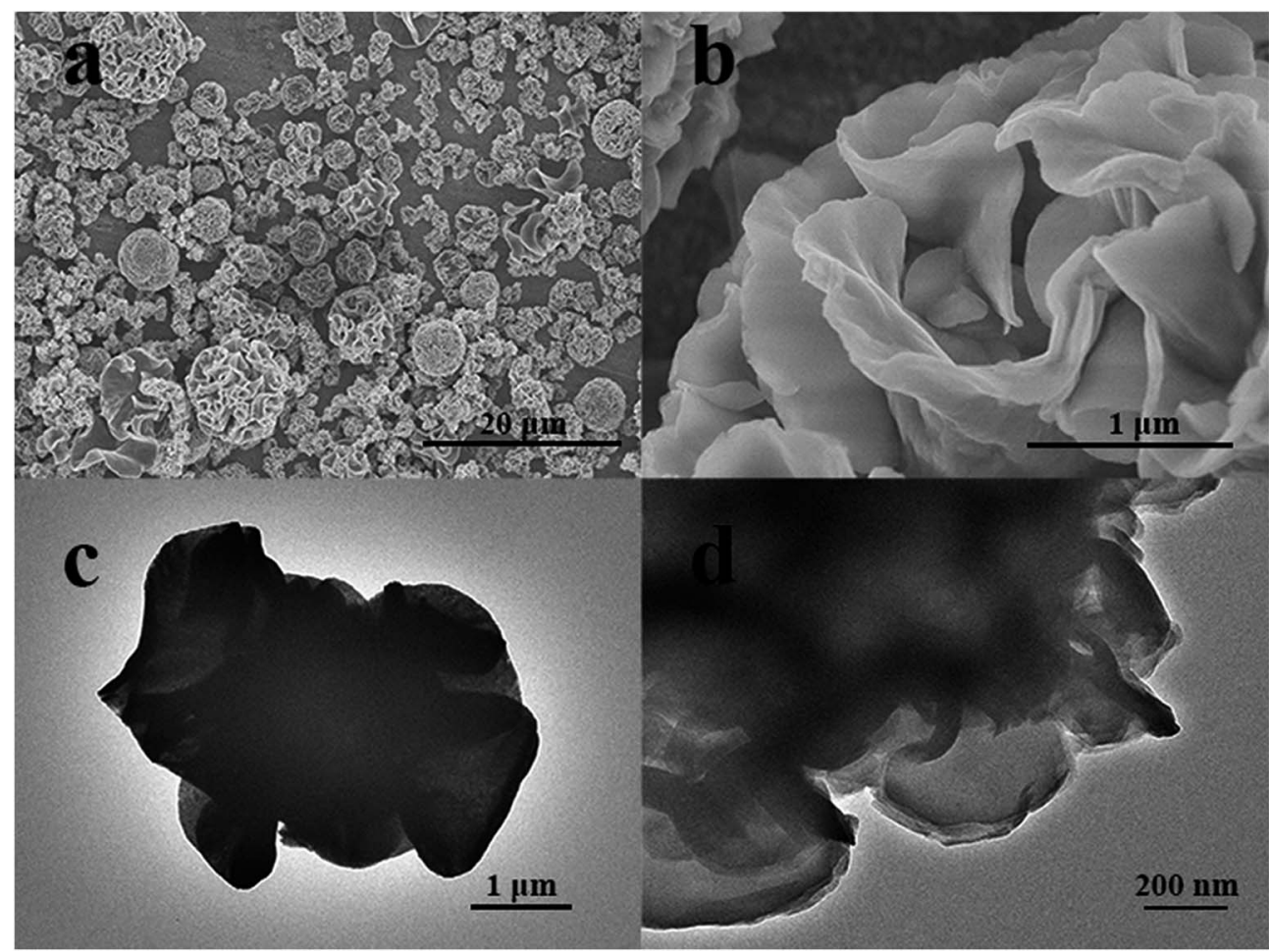

Fig. 3 SEM ( $a$ and b) and TEM ( $c$ and d) images of CuAl-LDH.

Fig. 4 presents the $\mathrm{N}_{2}$ adsorption-desorption isotherm of the CuAl-LDH sample, which is a type IV isotherm according to the IUPAC classification. The $\mathrm{H}_{3}$-type hysteresis loop demonstrates that there are large amounts of slit-shaped pore channels formed due to the aggregation of plate-like layers. Meanwhile, the corresponding pore size distribution curve shows a wide range of pore sizes, implying that the partial pore size extends into the macropore range. The BET surface area, pore diameter, and pore volume of the catalysts are summarized in Table 1 . The calculated surface area of the sample is $6.4772 \mathrm{~m}^{2} \mathrm{~g}^{-1}$. This result could be attributed to the decline in orderliness of the layered double hydroxide crystallite when there is a larger $\mathrm{Cu}$ component. This could also be explained by Jahn-Teller



Fig. $4 \quad \mathrm{~N}_{2}$ adsorption-desorption isotherm and pore size distribution curve of CuAl-LDH sample. distortion which brings about poor long range ordering. ICP and EA analysis of the hydrotalcite samples are shown in Table 2. The content of $\mathrm{Al}^{3+}$ is consistent with the theoretical composition. The $\mathrm{Cu}^{2+}$ content in $\mathrm{Cu} / \mathrm{Al}$ at $2.1: 1$ is slightly higher than expected. The $\mathrm{N}$ and $\mathrm{C}$ contents of the CuAl-LDH are $4.23 \%$ and $0.48 \%$, respectively, which indicates that the intercalated ions are mainly nitrate-based. A small amount of carbonates is derived from the decomposition of urea.

\subsection{Catalytic test of $\mathrm{Cu} / \mathrm{Al} \mathrm{LDH}$}

The catalytic performance of CuAl-LDH for the degradation of MO was measured at $200-800 \mathrm{~nm}$ using UV-visible spectroscopy. The degradation reactions were based on the hydroxyl radicals originating from peroxide degradation, which play a crucial role in the degradation of organic pollutants under near-neutral conditions. In order to enhance the reaction rate, the reaction conditions, such as the reaction temperature, the amount of catalyst and $\mathrm{H}_{2} \mathrm{O}_{2}$ dosages, have been optimized.

The effect of $\mathrm{H}_{2} \mathrm{O}_{2}$ dosage on the decomposition efficiency of methyl orange was investigated first. From the results in Fig. 5a, when the reaction temperature is $25^{\circ} \mathrm{C}, 20 \mathrm{mg}$ of CuAl-LDH is added to $50 \mathrm{~mL}$ of $20 \mathrm{mg} \mathrm{L}^{-1}$ methyl orange solution with varying amounts of $\mathrm{H}_{2} \mathrm{O}_{2}$. It can be seen that before the addition

Table 1 Textural parameters

\begin{tabular}{llll}
\hline Catalyst & $S_{\text {BET }}\left(\mathrm{m}^{2} \mathrm{~g}^{-1}\right)$ & $V_{\mathrm{P}}\left(\mathrm{cm}^{3} \mathrm{~g}^{-1}\right)$ & $D_{\mathrm{P}}(\mathrm{nm})$ \\
\hline CuAl-LDH & 6.4772 & 0.01918 & 7.3584
\end{tabular}


Table 2 Results of elemental analysis

\begin{tabular}{llllll}
\hline Catalyst & $\begin{array}{l}\text { Ratio of } \\
\mathrm{Cu}: \mathrm{Al}\end{array}$ & $\mathrm{Cu}(\mathrm{wt} \%)$ & $\mathrm{Al}(\mathrm{wt} \%)$ & $\mathrm{N}(\mathrm{wt} \%)$ & $\mathrm{C} \mathrm{(wt \% )}$ \\
\hline CuAl-LDH & $2.1: 1$ & 37.1 & 7.49 & 4.23 & 0.48
\end{tabular}

of $\mathrm{H}_{2} \mathrm{O}_{2}$, the change in $\mathrm{MO}$ concentration is due to the adsorption of methyl orange on the surface of the LDH materials. The amount of methyl orange adsorbed increases rapidly in the initial $10 \mathrm{~min}$. Thereafter, the adsorption rate becomes slower. This could be explained by the fact that more vacant adsorption sites are available in the initial stage and the electrostatic attraction also accelerates the adsorption of methyl orange on the surface of $\mathrm{CuAl}-\mathrm{LDH}$, but, after a period of time, the remaining vacant adsorption sites are difficult to occupy because of the repulsive forces between the solution molecules in the solid phases. ${ }^{41}$ Compare the blank experiment: when the $\mathrm{H}_{2} \mathrm{O}_{2}$ dosage increased to $0.5 \mathrm{~mL}$, the removal rate of methyl orange improved from $70.12 \%$ to $93.05 \%$ at $90 \mathrm{~min}$, and when the $\mathrm{H}_{2} \mathrm{O}_{2}$ dosage was increased further to $2 \mathrm{~mL}$, the value reached $99.04 \%$ within $50 \mathrm{~min}$. The higher the $\mathrm{H}_{2} \mathrm{O}_{2}$ dosage, the faster the increase in the removal of methyl orange. The above results indicate that the catalytic activity has been improved significantly with an increase in the $\mathrm{H}_{2} \mathrm{O}_{2}$ dosage, which is due to the interaction between $\mathrm{CuAl}-\mathrm{LDH}$ and $\mathrm{H}_{2} \mathrm{O}_{2}$ that generates more hydroxyl radicals, leading to the rapid decomposition of methyl orange.

We illustrate the influence of the amount of catalyst on methyl orange decolorization in the presence of $0.5 \mathrm{~mL} \mathrm{H}_{2} \mathrm{O}_{2}$ at $25{ }^{\circ} \mathrm{C}$. It can be seen that the concentration change in $\mathrm{MO}$ is imperceptible after treatment with the $\mathrm{H}_{2} \mathrm{O}_{2}$ without the addition of $\mathrm{CuAl}-\mathrm{LDH}$. With an increase in $\mathrm{CuAl}-\mathrm{LDH}$, the color removal efficiency significantly improved. As expected in Fig. 5b, when the dosage is increased to $20 \mathrm{mg}, 93 \%$ of the original concentration of the methyl orange solution has been removed over CuAl-LDH after $90 \mathrm{~min}$. When the dosage is increased from $20 \mathrm{mg}$ to $40 \mathrm{mg}$, the final degradation efficiency reaches $100 \%$ in 30 minutes, which means that the dosages are the main reason for the degradation. The results above should be attributed to the fact that more active sites can be contacted with hydrogen peroxide. Meanwhile, there are more adsorption sites which can also remove the methyl orange. Even though there is a synergy effect between the catalysis and the adsorption, the catalytic effect occupies the dominant position at the beginning of the initial stage. So the removal rate of methyl orange rapidly increases in a short time.

The influence of the reaction temperature on the MO degradation rate was also investigated. The results are given in Fig. $5 \mathrm{c}$ and $\mathrm{d}$. The dosages of $\mathrm{CuAl}-\mathrm{LDH}$ and the $\mathrm{H}_{2} \mathrm{O}_{2}$ were kept constant at $20 \mathrm{mg}$ and $0.5 \mathrm{~mL}$, separately, and it was observed that the degradation efficiency increased sharply and reached
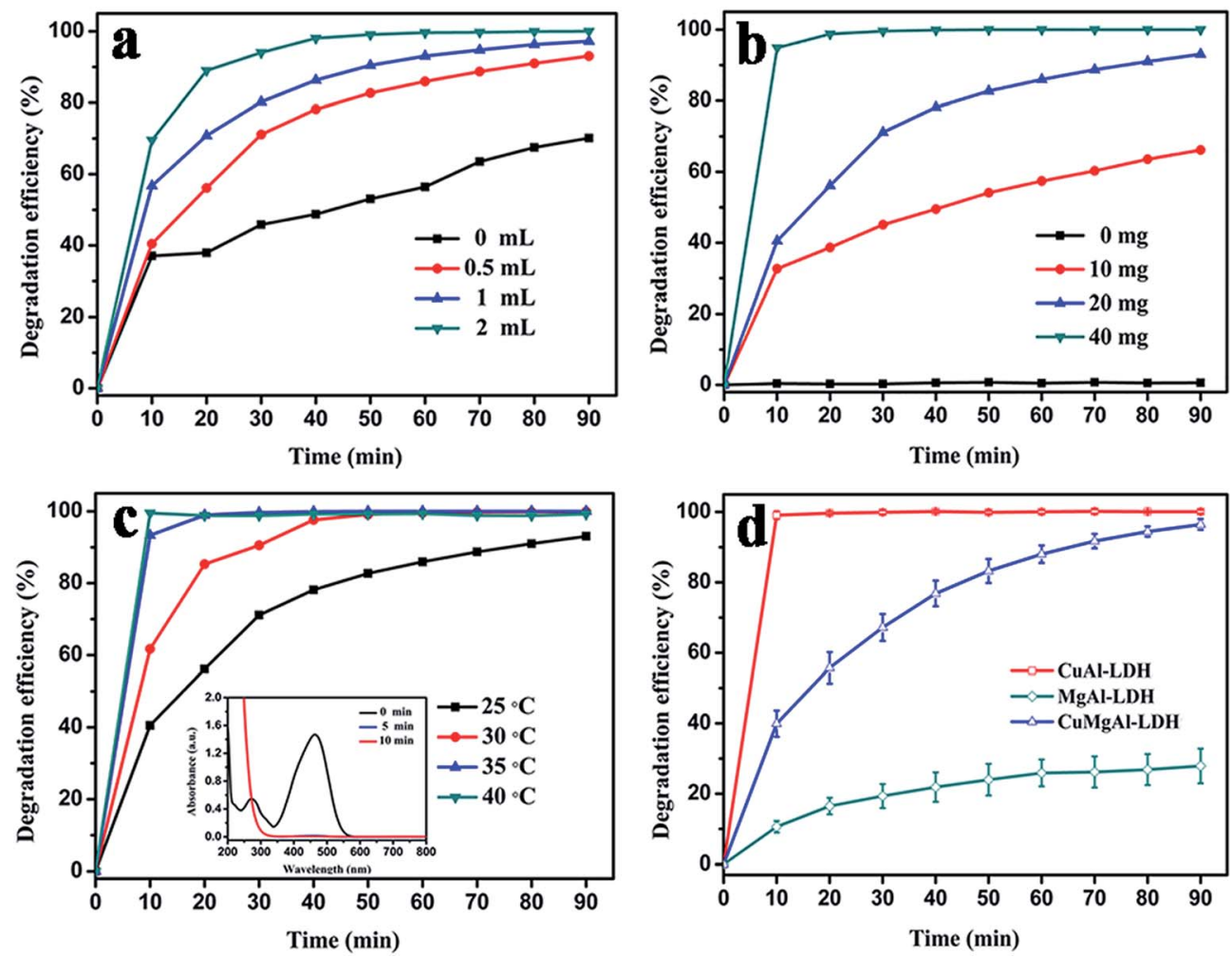

Fig. 5 (a) Effects of hydrogen peroxide dosages on $\mathrm{MO}$ degradation ([MO] $=20 \mathrm{mg} \mathrm{L}^{-1}$, [CuAl-LDH] $=20 \mathrm{mg}, T=25^{\circ} \mathrm{C}$ ). (b) Effects of catalyst dosages on $\mathrm{MO}$ degradation ( $[\mathrm{MO}]=20 \mathrm{mg} \mathrm{L}^{-1}$, $\left[\mathrm{H}_{2} \mathrm{O}_{2}\right]=0.5 \mathrm{~mL}, T=25^{\circ} \mathrm{C}$ ). (c) Effects of temperature on $\mathrm{MO}$ degradation ([MO] $=20 \mathrm{mg} \mathrm{L} \mathrm{L}^{-1}$, $\left[\mathrm{H}_{2} \mathrm{O}_{2}\right]=0.5 \mathrm{~mL}$, [CuAl-LDH] $=20 \mathrm{mg}$ ). (d) The degradation of $\mathrm{MO}$ by different types of layered double hydroxides ([MO] $=20 \mathrm{mg} \mathrm{L}{ }^{-1}$, [LDHs] $=$ $20 \mathrm{mg}$, and $\left[\mathrm{H}_{2} \mathrm{O}_{2}\right]=0.5 \mathrm{~mL}, T=40^{\circ} \mathrm{C}$ ). 
$100 \%$ after $5 \mathrm{~min}$ when the reaction temperature was elevated from $25{ }^{\circ} \mathrm{C}$ to $40{ }^{\circ} \mathrm{C}$. Two factors may be responsible for this increase in removal efficiency. Firstly, the catalytic performance increases significantly at high temperature, which is due to the fact that heating enhances the reaction rate of MO with hydrogen peroxide. Secondly, the higher temperature will decrease the adsorption effect and make full use of the activity sites produced by hydrogen peroxide. As a result, the MO is decomposed rapidly in a short time.

Different types of layered double hydroxides were used as catalysts to degrade MO, as shown in Fig. 5d. MgAl-LDHs have no good effect on the degradation of MO: the removal rate of MO is only $27.9 \%$ after $90 \mathrm{~min}$. The reason for the fading of MO is attributed to the poor catalytic activity of $\mathrm{MgAl}-\mathrm{LDH}$. When copper ions are introduced into the layered double hydroxides, the catalytic activity is significantly improved. We can see that the final degradation efficiency of MO reaches $96.5 \%$ over the MgAlCu-LDHs after $90 \mathrm{~min}$. Whereas, the degradation rate of MO has already reached $99.1 \%$ in 10 min with CuAl-LDH. This suggests that the degradation of MO is dependent on the catalytic effect of the copper ions. Meantime, the higher the copper ion content in the layered double hydroxides, the faster the degradation of MO.

\subsection{Cyclic utilization of CuAl-LDH}

Reutilization is one of the greatest advantages of a heterogeneous catalyst, which can also provide useful information about catalytic stability during the catalytic cycles. The recyclability of the CuAl-LDH catalyst was subsequently examined. The catalyst showed appreciable reduction in activity after every run. As shown in Fig. 6, the degradation ratio of MO by the $\mathrm{LDH}$ powder sample in the first cycle was $100 \%$, but it decreased very quickly and only $20.4 \%$ was obtained in the fifth cycle. The experiment indicates that after a certain number of reaction cycles, the catalytic effect decreased very significantly. The result can be attributed to MO and its decomposition of the intermediates adsorbed on the surface of the material, which hinder the hydrogen peroxide in making contact with the active site, leading to a decrease in the hydroxyl radical content.

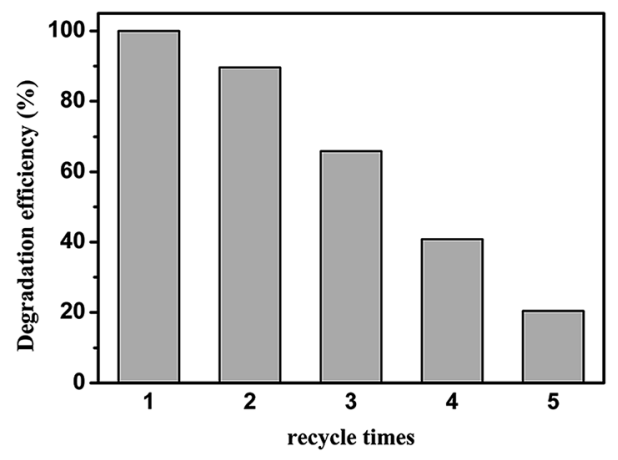

Fig. 6 Reusability of CuAl-LDH for the degradation of MO. All experiments for catalyst reuse were performed for $1.5 \mathrm{~h}$.

\section{Conclusions}

In summary, a flower-like CuAl-LDH with a high content of copper ions was successfully prepared through a solvothermal method with water and dimethyl sulfoxide as a mixed solvent. The as-prepared material was found to be an effective heterogeneous catalyst for MO removal, and it exhibited excellent catalytic activity under optimized conditions. In addition, the prepared catalysts exhibited excellent stability of their structural and catalytic properties.

\section{Acknowledgements}

Fund of University Students' Research and Innovation Projects of China and Shanghai (No. 201610856014, cs1501011, cs1604006).

\section{Notes and references}

1 P. Li, Y. Song, S. Wang, Z. Tao, S. Yu and Y. Liu, Ultrason. Sonochem., 2015, 22, 132-138.

2 J. Han, H. Y. Zeng, S. Xu, C. R. Chen and X. J. Liu, Appl. Catal., A, 2016, 527, 72-80.

3 C. Wang, F. Xing, Y. L. Bai, Y. Zhao, M. X. Li and S. Zhu, Cryst. Growth Des., 2016, 16, 2277-2288.

4 S. X. Yang, L. Y. Wang, X. D. Zhang, W. J. Yang and G. L. Song, Chem. Eng. J., 2015, 275, 315-321.

5 Y. B. Liu, L. Yu, C. N. Ong and J. P. Xie, Nano Res., 2016, 9, 1983-1993.

6 Y. B. Liu, Y. Y. Zheng, B. W. Du, R. R. Nasaruddin, T. K. Chen and J. P. Xie, Ind. Eng. Chem. Res., 2017, 56, 2999-3007.

7 Y. B. Liu, Q. F. Yao, X. J. Wu, T. k. Chen, Y. Ma, C. N. Ong and J. P. Xie, Nanoscale, 2016, 8, 10145-10151.

8 Y. B. Liu, J. P. Xie, C. N. Ong, C. D. Vecitis and Z. Zhou, Environ. Sci.: Water Res. Technol., 2015, 1, 769-778.

9 Y. B. Liu, H. Liu, Z. Zhou, T. R. Wang, C. N. Ong and C. D. Vecitis, Environ. Sci. Technol., 2015, 49, 7974-7980.

10 Y. B. Liu, J. H. D. Lee, Q. Xia, Y. Ma, Y. Yu, L. Y. L. Yung, J. P. Xie, C. N. Ong, C. D. Vecitis and Z. Zhou, J. Mater. Chem. A, 2014, 2, 16554-16562.

11 Y. Li and F. S. Zhang, Chem. Eng. J., 2010, 158, 148-153.

12 Y. Zhan, H. Li and Y. Chen, J. Hazard. Mater., 2010, 180, 481485.

13 D. A. Nichela, A. M. Berkovic, M. R. Costante, M. P. Juliarena and F. S. García Einschlag, Chem. Eng. J., 2013, 228, 1148-1157.

14 M. Munoz, Z. M. de Pedro, J. A. Casas and J. J. Rodriguez, Appl. Catal., B, 2015, 176-177, 249-265.

15 Y. Wang, Y. Gao, L. Chen and H. Zhang, Catal. Today, 2015, 252, 107-112.

16 M. Dukkanci, G. Gunduz, S. Yilmaz and R. V. Prihod'ko, J. Hazard. Mater., 2010, 181, 343-350.

17 L. Lyu, L. Zhang and C. Hu, Chem. Eng. J., 2015, 274, 298306.

18 A. D. Bokare and W. Choi, J. Hazard. Mater., 2014, 275, 121135.

19 C. Li, M. Wei, D. G. Evans and X. Duan, Catal. Today, 2015, 247, 163-169. 
20 C. Li, M. Wei, D. G. Evans and X. Duan, Small, 2014, 10, 4469-4486.

21 S. He, Z. An, M. Wei, D. G. Evans and X. Duan, Chem. Commun., 2013, 49, 5912-5920.

22 Z. P. Xu, J. Zhang, M. O. Adebajo, H. Zhang and C. Zhou, Appl. Clay Sci., 2011, 53, 139-150.

23 G. Fan, F. Li, D. G. Evans and X. Duan, Chem. Soc. Rev., 2014, 43, 7040-7066.

24 T. Baskaran, J. Christopher and A. Sakthivel, RSC Adv., 2015, 5, 98853-98875.

25 S. Kannan, A. Dubey and H. Knozinger, J. Catal., 2005, 231, 381-392.

26 J. Fahel, S. Kim, P. Durand, E. Andre and C. Carteret, Dalton Trans., 2016, 45, 8224-8235.

27 H. Lu, Z. Zhu, H. Zhang, J. Zhu, Y. Qiu, L. Zhu and S. Kuppers, ACS Appl. Mater. Interfaces, 2016, 8, 2534325352.

28 G. Cui, F. Wang, S. He and M. Wei, RSC Adv., 2016, 6, 105406-105411.

29 H. Zhang, G. Zhang, X. Bi and X. Chen, J. Mater. Chem. A, 2013, 1, 5934-5942.

30 M. G. Churchil, A. Antonyraj and S. Kannan, Ind. Eng. Chem. Res., 2010, 49, 6020-6026.
31 M. Intissar, A. Seron, F. Giovannelli, C. Autret, M. MotelicaHeino and F. Delorme, J. Mater. Sci., 2014, 50, 1427-1434.

32 F. M. A. Alejandre, X. Rodriguez, P. Salagre, Y. Cesteros and J. E. Sueiras, Appl. Catal., B, 2001, 30, 195-207.

33 N. Tien Thao and L. T. Kim Huyen, Chem. Eng. J., 2015, 279, 840-850.

34 B. Li, Y. Zhao, S. Zhang, W. Gao and M. Wei, ACS Appl. Mater. Interfaces, 2013, 5, 10233-10239.

35 P. Ma, Z. Xiao, S. Jin, C. Li and C. Liang, J. Energy Chem., 2016, 25, 782-792.

36 L. H. Zhang, F. Li, D. G. Evans and X. Duan, Ind. Eng. Chem. Res., 2010, 49, 5959-5968.

37 J. T. Kloprogge, L. Hickey and R. L. Frost, J. Raman Spectrosc., 2004, 35, 967-974.

38 J. T. Kloprogge, D. Wharton, L. Hickey and R. L. Frost, Am. Mineral., 2002, 87, 623-629.

39 V. Rives, A. Dubey and S. Kannan, Phys. Chem. Chem. Phys., 2001, 3, 4826-4836.

40 L. Dou and H. Zhang, J. Mater. Chem. A, 2016, 4, 1899019002.

41 Z. Yang, S. Ji, W. Gao, C. Zhang, L. Ren, W. W. Tjiu, Z. Zhang, J. Pan and T. Liu, J. Colloid Interface Sci., 2013, 408, 25-32. 